\title{
The impact of COVID-19 pandemic on glycemic control in patients with diabetes mellitus in Turkey: a multi-center study from Kocaeli
}

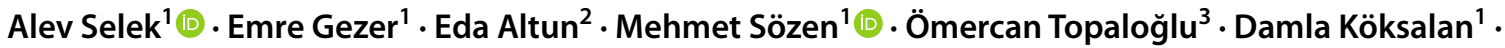 \\ Halil Demirkan ${ }^{4} \cdot$ Dilek Karakaya $^{5} \cdot$ Berrin Cetinarslan $^{1} \cdot$ Zeynep Cantürk $^{1} \cdot$ Dilek Taymez $^{6}$
}

Received: 6 July 2021 / Accepted: 21 August 2021 / Published online: 27 August 2021

(c) Springer Nature Switzerland AG 2021

\begin{abstract}
Aims The aim of this study is to determine the impact of the COVID-19 pandemic on the management and disease control of the patients with diabetes mellitus in Kocaeli.

Methods This study was carried out in six leading central hospitals in five major districts of Kocaeli. The study was conducted between June 2020 and November 2020. The patients who had previous admissions to these clinics within 6 months prior to the pandemic were enrolled in the study.

Results A total of 283 patients were enrolled in the study, among them 151 (53\%) patients were female, 268 (95\%) had type $2 \mathrm{DM}$ and remaining $5 \%$ had type $1 \mathrm{DM}$. The median weight of the patients was similar between the previous and last visits ( $84 \mathrm{~kg}$ vs $83 \mathrm{~kg}, \mathrm{p}=0,88$ ). Laboratory parameters of previous and current visits revealed that mean fasting plasma glucose (FPG) and HbA1c levels were not significantly changed. The number of the patients who had controlled blood pressure was significantly decreased. The number of those who had neuropathic complains and the severity of dyslipidemia significantly increased during pandemic period.

Conclusion Our study demonstrated that despite decreased compliance with diet and exercise, and difficulty in accessing medication, there was no significant change in weight, FPG and HbA1c levels in diabetic patients. Since cultural differences, education level and socioeconomic opportunities differ between societies, national and international studies will be more accurate to evaluate the effects of epidemics on the course of chronic diseases.
\end{abstract}

Keywords COVID-19 $\cdot$ Pandemic $\cdot$ Diabetes mellitus $\cdot \mathrm{HbA1c}$

\section{Introduction}

The COVID-19 pandemic continues to impact the world since the beginning of 2020. It has spread all over the world and been threatening the lives of millions. Turkey has been one of the most affected countries by the COVID-19. According to the data of the World Health Organization in March, Turkey is among the top 10 countries most affected by COVID-19 with approximately 2.8 million affected cases [1]. Following the first officially notified case in Turkey on March $11^{\text {th }} 2020$, a lockdown policy had been started. The status of social restriction has been arranged according to the

iThenticate plagiarism result: $\% 15$

Mehmet Sözen

mehmetsozen07@gmail.com

Extended author information available on the last page of the article rate of case detection. Kocaeli is one of the most crowded cities of Turkey and a high-risk area for COVID-19, resulting in stricter social restrictions. In this situation, routine care for patients with chronic diseases has become a big challenge.

The COVID-19 pandemic has negatively impacted health care of people with chronic diseases all over the world. The social restrictions, patients' concerns, inadequate health care systems and even COVID-19 per se resulted in deterioration in chronic diseases. Diabetes mellitus (DM) is one of the leading chronic diseases. The latest edition of the IDF Diabetes Atlas shows that 463 million adults are currently living with diabetes [2]. Diabetes is a common comorbidity in patients with COVID-19 and also associated with higher mortality rates in hospitalized patients [3,4]. On the other hand, it can be predicted that diabetic patients without COVID-19 may also be affected by the pandemic for several reasons. The management of diabetes mainly depends on the 
patients' compliance and self-control. Compliance with life style modification, medical treatment and follow up visits are important predictors of success in the management of diabetic patients. It has been shown that Diabetes was the most impacted chronic disease by the reduction in access to a medical care during pandemic [5]. A web-based survey from the United States showed that the prevalence of any delay or avoidance of medical care was $31.5 \%$ in routine outpatient care [6].

Social restrictions were very strict at the beginning and during the second peak of pandemic in Kocaeli. Three leading hospitals and the university hospital of the city became pandemic hospitals; therefore elective healthcare appointments were either cancelled or postponed. There was a curfew for everyone on weekends and also all week days for elderly. All indoor sports areas and pools were closed. These restrictions resulted in a reduction in physical activity which is one of the cornerstones of life style modification treatment of diabetes. It can be predicted that, these unfavorable conditions may adversely affect diabetes management; however, there is limited data in the literature with this regard. The aim of this study is to determine the impact of the COVID19 pandemic on the management and disease control of the patients with diabetes mellitus in Kocaeli.

\section{Materials and methods}

\section{Study design and population}

This study was carried out in six leading central hospitals in five major districts of Kocaeli. Study centers included a university hospital, a training and research hospital and four state hospitals. All authors in the study were specialist for the treatment of diabetes.

The study was conducted between June 2020-November 2020; after the resolution of the first peak of COVID-19 and during the first normalization period in Turkey. All patients who were admitted to the study clinics with the diagnosis of diabetes mellitus were evaluated. The patients who had previous admissions to these clinics within 6 months prior to the pandemic were enrolled in the study. The inclusion and exclusion criteria are as follows;

Inclusion criteria;

1. Patients $>18$ years of age

2. Being under follow-up and treatment with the diagnosis of Type 1 or Type 2 Diabetes for at least 6 months

Exclusion criteria;

\section{Previous COVID 19}

2. Severe infection, surgery or any other systemic disease activation after last follow up visit

3. Active malignancy

4. Pregnancy

5. Any antidiabetic medication change in the last 3 months

6. Use of any drugs that may acutely affect blood glucose levels, such as corticosteroids, cyclosporine

7. Decompensated liver disease and heart failure

After exclusion of patients having specified exclusion criteria, 686 patients were eligible. Unfortunately, only 283 patients could be enrolled to the study who had previous admissions to the study clinics within 6 months just prior to the pandemic and given written informed consent. Remaining 403 patients were either on first admission or previous admission was before $>6$ months or not given written informed consent.

Demographic features, comorbid diseases (hypertension (HT), hyperlipidemia (HL), chronic kidney disease, coronary artery disease and cerebrovascular disease), current antidiabetic medications, diet and exercise program adherence, hypo/hyperglycemic episodes, neuropathic symptoms, and presence of emotional stress were noted. Hypo/hyperglycemic episodes were defined according to patients' self-monitoring of capillary blood glucose reports $(<70 \mathrm{mg} / \mathrm{dl}$ for hypoglycemia, $>250 \mathrm{mg} / \mathrm{dl}$ for hyperglycemia). Compliance with diet and exercise was assessed according to patients self-report and categorized into 3 levels, i.e., poor, moderate and good. Difficulties in accessing medical assistance and medication were also questioned. Weight and body mass index (BMI) were measured. Self-assessed fasting and post prandial capillary glucose levels, $\mathrm{HbA1c}$, fasting plasma glucose and cholesterol levels were noted. All these parameters were also evaluated from the records of previous outpatient clinic visits in order to compare the findings before and after pandemic.

\section{Statistical analysis}

All statistical analyses were performed using the IBM SPSS for Windows version 20.0 (IBM Corp., Armonk, NY, USA). The Kolmogorov-Smirnov test was used to test the normality of data distribution. Continuous variables were expressed as median $\left(25^{\text {th }}-75^{\text {th }}\right.$ percentiles), and categorical variables were expressed as counts (percentages). Comparisons of nonnormally distributed continuous variables between the times were performed using the Wilcoxon Signed Ranks Test. Comparisons of categorical variables between the times were performed using the Mc Nemar Chi Square test. A two-sided $\mathrm{P}$ value $<0.05$ was considered statistically significant. 
Table 1 Patient's characteristics

\begin{tabular}{ll}
\hline Parameters $(\mathrm{n}=283)$ & Value $\mathrm{n}(\%)$ \\
\hline Age (years) & $58.0 \pm 12.1$ \\
Male/female & $132(47 \%) / 151(53 \%)$ \\
Duration of diabetes, years & $11 \pm 6$ \\
DM Type & \\
Type 1 & $15(5 \%)$ \\
Type 2 & $268(95 \%)$ \\
Comorbid diseases & \\
Hypertension & $200(70 \%)$ \\
Hyperlipidemia & $186(66 \%)$ \\
Chronic kidney disease & $108(38 \%)$ \\
Coronary artery disease & $81(29 \%)$ \\
Cerebrovascular disease & $17(6 \%)$ \\
Pharmacotherapy type for DM & \\
Dipeptidyl peptidase-4 inhibitors & $130(46 \%)$ \\
Metformin & $158(56 \%)$ \\
Sulfonylurea & $32(11 \%)$ \\
Sodium-glucose cotransporter-2 inhibi- & $90(32 \%)$ \\
tors & \\
Pioglitazone & $17(6 \%)$ \\
Glucagon-like peptide-1 receptor agonist & $56(20 \%)$ \\
Insulin & $148(52 \%)$ \\
Basal insulin & $71(25 \%)$ \\
$\quad$ Mixed insulin & $28(10 \%)$ \\
Intensive insulin & $49(17 \%)$ \\
Number of oral antidiabetic drug type & \\
None & $41(14 \%)$ \\
One & $107(38 \%)$ \\
Two & $88(31 \%)$ \\
\hline
\end{tabular}

\section{Results}

A total of 283 patients were enrolled in the study, among them 151 (53\%) patients were female, 268 (95\%) had type $2 \mathrm{DM}$ and remaining $5 \%$ had type $1 \mathrm{DM}$. The median age was $58 \pm 12$ and similar in both genders, median time for the diagnosis of DM was $12 \pm 4$ years. Concerning comorbid diseases, $200(70 \%)$ patients had HT, 186 (66\%) had HL, 108 (38\%) had chronic kidney disease, 81 (29\%) had coronary artery disease and 17 (6\%) had cerebrovascular disease. Among 200 patients with HT, 186 (93\%) of them were using antihypertensive treatment and $78 \%$ were on target ranges for blood pressure. $141(78 \%)$ of the patients with HL were using statins but mean LDL levels were above target limits (Table 1).

Evaluation of antidiabetic medication revealed that 41 $(14 \%)$ of patients were not using any antidiabetic medication. Remaining 241 patients were using at least one oral antidiabetic drug (OAD), among them 107 (38\%) patients were using one OAD, 88 (31\%) 2 OADs and $47(17 \%) \geq 3$ OADs. $148(52 \%)$ patients were on insulin treatment. The details of antidiabetic medications were shown in Table 1.

Comparison of clinical findings on previous and current visits (Table 2) revealed that, the number of the patients who had poorly controlled blood pressure was significantly increased $(22 \%$ vs $27 \% \mathrm{p}<0.001)$. The number of the patients that experienced hypo or hyperglycemic episodes, detected with self-glucose monitoring, was similar at both previous and current visits. The patients having neuropathic complains were significantly higher at the current visit then the previous one ( $31 \%$ vs. $42 \%, \mathrm{p}<0.001$ ). Compliance with diet and exercise was categorized into 3 levels, i.e., poor, moderate and good. The number of the patients with poor diet and exercise compliance was significantly increased at the current visit then the previous one $(p=0.003, p<0.001$ respectively). Difficulty in accessing medication was reported by 19 (7\%) patients at the current visit, $3(1 \%)$ at the previous visit $(\mathrm{p}=0.001)$. The number of the patients that report difficulty in accessing to medical assistance from health care professionals, increased from
Table 2 Changes in clinical parameters before and after lockdown

\begin{tabular}{lllc}
\hline Parameters & $\begin{array}{l}\text { Before lockdown } \\
\mathrm{n}(\%)\end{array}$ & $\begin{array}{l}\text { After lockdown } \\
\mathrm{n}(\%)\end{array}$ & $\mathrm{p}$ value \\
\hline Body weight $(\mathrm{kg})( \pm \mathrm{SD})$ & $84( \pm 17)$ & $83( \pm 18)$ & 0.88 \\
Poorly controlled hypertension & $58(22 \%)$ & $71(27 \%)$ & $<0.001$ \\
Hypoglycemic episodes & $26(10 \%)$ & $34(12 \%)$ & 0.24 \\
Hyperglycemic episodes & $22(8 \%)$ & $18(7 \%)$ & 0.45 \\
Neuropathic complains & $85(31 \%)$ & $114(42 \%)$ & $<0.001$ \\
Poor diet compliance & $70(26 \%)$ & $96(35 \%)$ & 0.003 \\
Poor exercise compliance & $101(37 \%)$ & $162(58 \%)$ & $<0.001$ \\
Difficulty in accessing medication & $3(1 \%)$ & $19(7 \%)$ & $<0.001$ \\
Difficulty in accessing to medical assistance & $2(1 \%)$ & $54(19 \%)$ & $<0.001$ \\
Complaining of emotional stress & $38(13 \%)$ & $143(50 \%)$ & $<0.001$ \\
\hline
\end{tabular}


$1 \%$ to $\% 19$ between 2 visits $(\mathrm{p}=0.001)$. Complaining of emotional stress was seen in $38(13 \%)$ patients at the previous visit while increased to $143(50 \%)$ patients at the current visit $(\mathrm{p}<0.0001)$.

The median weight of the patients was similar between the previous and last visits ( $84 \mathrm{~kg}$ vs $83 \mathrm{~kg}, \mathrm{p}=0,88$ ). Laboratory parameters of previous and current visits revealed that mean fasting plasma glucose (FPG) and $\mathrm{HbA} 1 \mathrm{c}$ levels were similar but fasting triglyceride $(179 \mathrm{mg} / \mathrm{dL}$ vs $194 \mathrm{mg} / \mathrm{dL})$ and LDL $(125 \mathrm{mg} / \mathrm{dL}$ vs $144 \mathrm{mg} / \mathrm{dL}$ ) levels were significantly increased between the visits (Table 3 ). The evaluation of type 1 diabetic patients showed similar results with the entire cohort. FPG and HbA1c levels were not changed ( $\mathrm{p}=0.94$ and 0.28 , respectively) (Table 3 ).

\section{Discussion}

The COVID-19 pandemic has impacted the whole world for several aspects, of which health care systems have been affected the most. Disease burden of COVID-19 negatively impacted health care providers, hospital facilities and even drug and medical equipment supply. On the other hand, social restrictions, lockdown policies and the concerns of patients resulted in a deterioration in the courses of several chronic diseases [7-10]. As DM is one of the leading chronic diseases, current study aimed to evaluate the effect of COVID-19 pandemic on the course of DM in patients without a COVID-19 history at any time. Our results showed that although diet and exercise adherence of the patients were decreased after pandemic, weight, FPG and HbA1c levels were not changed. This result was surprising, but might be related to some issues. This study was conducted in six leading central hospitals in five major districts of Kocaeli. All of the physicians who carried out the study were specialist for the treatment of diabetes mellitus. Mean HbA1c levels were nearly on target both at the previous (7.1\%) and current (7.2\%) visits, which may have been caused by a selection bias as the patients followed by these centers might have been controlled tighter and educated better for the disease management. It is well known that patient education and self-care promotion have a key role in the proper management of chronic illnesses [11]. A similar single center study by Onmez et al. was conducted in a geographically close city to Kocaeli [9]. They have shown that HbA1c levels after 75-day lockdown were increased from 7.67 to 8.11, although statistically not significant. Their study population included patients followed by a family medicine physician, 2 internists and an endocrinologist, while almost all of our study team consists of endocrinologists. Another issue might be the time span; we enrolled the patients who had previous attendance to the study clinics within 6 months prior to the pandemic. This period might not be enough to see a significant change in HbA1c levels, as Onmez et al. also concluded [9]. FPG levels were also not changed in our study, which was consistent with $\mathrm{HbA1c}$ levels.

There are many studies in different countries evaluating type 1 and type 2 diabetic patients before and after lockdown, but the results were controversial. Many studies conducted on type 1 diabetic patients showed an improvement in glycemic control. Indeed, these patients were using continuous glucose monitoring (CGM), therefore improvement in glycemic control may be due to improved disease self-management and getting more time for diabetes management [12-15]. However, Verma et al. showed that glycemic control worsened in patients with type 1 diabetes who were not using CGM [16]. In our study, the number of patients with type $1 \mathrm{DM}$ was relatively low and only 2 of them were using CGM. This may be an explanation for the stability of $\mathrm{HbA} 1 \mathrm{c}$ and FPG levels in type 1 diabetic patients.

Data concerning patients with type $2 \mathrm{DM}$ are more sophisticated. Two studies from different regions of Japan revealed opposite results. Tanji et al. concluded that $\mathrm{HbA} 1 \mathrm{c}$ levels were increased after the state of emergency; however Masuda et al. noted a significant decrease in HbAlc levels in both type 1 and type 2 DM. Indian, Chinese and Korean groups showed similar results. The authors revealed a deterioration in glycemic control of patients after pandemic [17-19]. In contrast, Psoma et al. from Greece showed improved glycemic control [20]. Furthermore, our study and some other studies showed that glycemic control was not changed [9, 21, 22].

The variations of the findings may be related several factors; such as, cultural difference, educational level, socioeconomic status, life style changes and health care facilities. In our country, CGM is not reimbursed, which makes disease
Table 3 Changes in laboratory parameters before and after lockdown

\begin{tabular}{lllcc}
\hline Parameters & Before lockdown & After lockdown & p value & Z value \\
\hline HbA1c (\%) & $7.1( \pm 1.7)$ & $7.2( \pm 5.2)$ & 0.97 & -0.03 \\
Fasting plasma glucose, mg/dl & $141( \pm 72)$ & $140( \pm 73)$ & 0.58 & -0.56 \\
Triglyceride, mg/dl & $169( \pm 100)$ & $194( \pm 132)$ & 0.02 & -2.33 \\
LDL, mg/dl & $125( \pm 62)$ & $144( \pm 40)$ & $<0.001$ & -3.80 \\
HDL, mg/dl & $42( \pm 12)$ & $44( \pm 11)$ & 0.31 & -1.00 \\
\hline
\end{tabular}


self-management difficult. The referral system is not mandatory in our country; therefore, patients can directly reach to an endocrinologist, this would positively affect the glycemic control. Our results showed that the difficulty in accessing medication was significantly increased (1\% vs $7 \%$ ); however, only 19 of the patients were complaining of the difficulty in accessing medication during lockdown. As a government policy, all medications which were previously prescribed by physicians for the treatment of chronic illnesses can be easily obtained from pharmacies without a prescription during pandemic period. This policy decreased unnecessary hospital attendance for only prescription and the difficulty in accessing medication. Otherwise, there would be more patients having difficulty in accessing to a medical assistance from health care professionals in our study.

Lockdown can clearly impact the lifestyle of the population, especially in terms of diet and physical activity. The diet and exercise compliance of the patients in our study were significantly decreased, in line with the most of the studies in literature [11, 23-25]. Studies from Spain, Italy, Poland and Japan showed that dietary habits and physical activity of the patients were changed. Mediterranean type diet compliance decreased, and consumption of frozen foods and alcohol increased. Physical activity also decreased, resulting in positive weight balance in some studies. However, even though dietary and exercise habits were changed negatively, weight gain was not detected in our study, similar to some studies in the literature $[9,22,26,27]$. The shortness of the study period may be a reason, why weight balance has not changed.

Furthermore, COVID-19 pandemic has also increased feelings of stress or anxiety in these patients, since they have been considered a high-risk group for COVID-19. Additionally, lockdown, social restrictions and economic burden of these policies would be some other leading causes. Increased levels of depression, anxiety, stress and negative affect were seen in several studies [28-30]. We evaluated the emotional stress from the patients self-declaration in our study, and the difference was very significant and dramatic, which might indicate that it should be evaluated further with questionnaires and scales.

Hypertension and hyperlipidemia were the most common co-morbidities in our study; nearly $3 / 4$ of the patients were complaining of HT and/or HL. COVID-19 pandemic also negatively impacted the control of HT and HL in our study; the percentage of the patients with uncontrolled HT and HL significantly increased after lockdown and restrictions. Our results were consistent with the situation of diet and exercise adherence of our cohort. Poor diet and exercise adherence and increased emotional stress would be the reasons of uncontrolled HT. The studies in hypertensive patients in the literature have demonstrated controversial results; showing both improved and worsened course of HT after lockdown period [31, 32].

There are several limitations of our study, of which including the patients who were followed-up by only endocrinologists, but not any physicians of family medicine nor internal medicine, might lead to a sampling bias. Relatively small sample size may be another limitation; however, we believe that 283 diabetic patients from 6 different leading hospitals are enough to represent a local data. In addition to those, study period was relatively short. Under pandemic circumstances, the literature regarding the related pandemic needs to be improved faster, compared to the regular period, in order to overcome the pandemic. Thus, we aimed to contribute to the literature without any delay by comparing the patients' data before and after the first peak of the COVID-19 pandemic.

\section{Conclusion}

In conclusion, our study demonstrated that despite decreased compliance with diet and exercise, and difficulty in accessing medication, there was no significant change in weight, FPG and HbA1c levels in diabetic patients. Moreover, the number of the patients who had controlled blood pressure decreased. The number of those who had neuropathic complains and the severity of dyslipidemia significantly increased during pandemic period. Since cultural difference, educational level, socioeconomic status, life style changes and health care facilities differ between societies, the data regarding the impact of pandemic on the courses of chronic diseases vary in the literature. As a result, national and even local investigations would be more accurate to evaluate the effects of pandemics on the courses of chronic illnesses.

Author Contributions A.S., B.C., Z.C., M.S., D.K., E.G. conceived and conducted the study. M.S., D.K., E.G, E.A., Ö.T., H.D., D.T., and D.M. involved the patients and do clinical visitis and follow-up. A.S., B.C., Z.C., M.S., D.K., E.G. contributed to the interpretation of the results. A.S. took the lead in writing the manuscript with the help of E.G. All authors provided critical feedback and helped shape the research, analysis and manuscript.

All authors read and approved the final version of the manuscript.

Funding This study was not financially supported by any institution or organization.

Availability of data and material The data sets analyzed during the current study are not publicly available but are available from the corresponding author on reasonable request.

Code availability Not applicable. 


\section{Declarations}

Ethics approval Ethical approval was obtained from the human research ethics committee of Kocaeli University Faculty of Medicine (2020/141), and permission for the research was obtained from the Turkish Republic Ministry of Health (2020-05-09T21-54-11). All the procedures complied with the provisions of the Declaration of Helsinki. All participants provided written informed consent.

Consent to participate Consent has been obtained from each patient or subject after full explanation of the purpose and nature of all procedures used.

Consent for publication Consent has been obtained from each patient or subject after full explanation of the purpose and nature of all procedures used.

Conflicts of interest/Competing interests The authors declare that they have no conflict of interest.

\section{References}

1. WHO. WHO Coronavirus (COVID-19) Dashboard. WHO Coronavirus (COVID-19) Dashboard With Vaccination Data. Who. 2021. p. 1-5.

2. Duke, L., Fereira de Moura, A., de Lapertosa, S. et al. IDF Diabetes Atlas 9th edition 2019. Int. Diabetes Fed. Diabetes Atlas, Ninth Ed. 2019.

3. Li B, Yang J, Zhao F, Zhi L, Wang X, Liu L, et al. Prevalence and impact of cardiovascular metabolic diseases on COVID-19 in China. Clin. Res. Cardiol. Springer; 2020. p. 531-8.

4. Kumar A, Arora A, Sharma P, Anikhindi SA, Bansal N, Singla V, et al. Is diabetes mellitus associated with mortality and severity of COVID-19? A meta-analysis. Diabetes Metab Syndr Clin Res Rev. Elsevier Ltd; 2020;14:535-45.

5. Chudasama Y V., Gillies CL, Zaccardi F, Coles B, Davies MJ, Seidu S, et al. Impact of COVID-19 on routine care for chronic diseases: A global survey of views from healthcare professionals. Diabetes Metab Syndr Clin Res Rev. Elsevier Ltd; 2020;14:965-7.

6. Czeisler MÉ, Marynak K, Clarke KEN, Salah Z, Shakya I, Thierry JM, et al. Delay or Avoidance of Medical Care Because of COVID-19-Related Concerns - United States, June 2020. MMWR Morb Mortal Wkly Rep. Centers for Disease Control MMWR Office; 2020;69:1250-7.

7. Manacorda T, Bandiera P, Terzuoli F, Ponzio M, Brichetto G, Zaratin $\mathrm{P}$, et al. Impact of the COVID-19 pandemic on persons with multiple sclerosis: Early findings from a survey on disruptions in care and self-reported outcomes. J Heal Serv Res Policy. SAGE Publications Ltd; 2020;

8. Kocatürk E, Salman A, Cherrez-Ojeda I, Criado PR, Peter J, Comert-Ozer E, et al. The global impact of the COVID-19 pandemic on the management and course of chronic urticaria. Allergy Eur J Allergy Clin Immunol. Blackwell Publishing Ltd; 2021;76:816-30.

9. Önmez A, Gamsızkan Z, Özdemir Ş, Kesikbaş E, Gökosmanoğlu F, Torun S, et al. The effect of COVID-19 lockdown on glycemic control in patients with type 2 diabetes mellitus in Turkey. Diabetes Metab Syndr Clin Res Rev. Elsevier Ltd; 2020;14:1963-6.

10. Kayikcioglu M, Kuman Tuncel O, Tokgozoglu L. Impact of the COVID-19 pandemic in patients with a previous history of premature myocardial infarction. Am J Prev Cardiol. Elsevier BV; 2020;4:100128.
11. Rees S, Williams A. Promoting and supporting self-management for adults living in the community with physical chronic illness: A systematic review of the effectiveness and meaningfulness of the patient-practitioner encounter. JBI Database Syst Rev Implement Reports. Ovid Technologies (Wolters Kluwer Health); 2009;7:492-582.

12. Fernández E, Cortazar A, Bellido V. Impact of COVID-19 lockdown on glycemic control in patients with type 1 diabetes. Diabetes Res Clin Pract. Elsevier Ireland Ltd; 2020;166.

13. Potier L, Hansel B, Larger E, Gautier JF, Carreira D, Assemien R, et al. Stay-at-home orders during the covid-19 pandemic, an opportunity to improve glucose control through behavioral changes in type 1 diabetes. Diabetes Care. American Diabetes Association Inc.; 2021;44:839-43.

14. Aragona M, Rodia C, Bertolotto A, Campi F, Coppelli A, Giannarelli $\mathrm{R}$, et al. Type 1 diabetes and COVID-19: The "lockdown effect.” Diabetes Res Clin Pract. Elsevier Ireland Ltd; 2020;170.

15. Christoforidis A, Kavoura E, Nemtsa A, Pappa K, Dimitriadou M. Coronavirus lockdown effect on type 1 diabetes management on children wearing insulin pump equipped with continuous glucose monitoring system. Diabetes Res Clin Pract. Elsevier Ireland Ltd; 2020;166.

16. Verma A, Rajput R, Verma S, Balania VKB, Jangra B. Impact of lockdown in COVID 19 on glycemic control in patients with type 1 Diabetes Mellitus. Diabetes Metab Syndr Clin Res Rev. Elsevier Ltd; 2020;14:1213-6.

17. Khare J, Jindal S. Observational study on Effect of Lock Down due to COVID 19 on glycemic control in patients with Diabetes: Experience from Central India. Diabetes Metab Syndr Clin Res Rev. Elsevier Ltd; 2020;14:1571-4.

18. Xue T, Li Q, Zhang Q, Lin W, Weng J, Li L, et al. Blood Glucose Levels in Elderly Subjects with Type 2 Diabetes During COVID19 Outbreak: A Retrospective Study in a Single Center. SSRN Electron J. Elsevier BV; 2020.

19. Park SD, Kim SW, Moon JS, Lee YY, Cho NH, Lee JH, et al. Impact of social distancing due to coronavirus disease 2019 on the changes in glycosylated hemoglobin level in people with type 2 diabetes mellitus. Diabetes Metab J. Korean Diabetes Association; 2021;45:109-14.

20. Psoma O, Papachristoforou E, Kountouri A, Balampanis K, Stergiou A, Lambadiari V, et al. Effect of COVID-19-associated lockdown on the metabolic control of patients with type 2 diabetes. J. Diabetes Complications. Elsevier Inc.; 2020.

21. Biancalana E, Parolini F, Mengozzi A, Solini A. Short-term impact of COVID-19 lockdown on metabolic control of patients with well-controlled type 2 diabetes: a single-centre observational study. Acta Diabetol. Springer-Verlag Italia s.r.1.; 2020;58.

22. Bonora BM, Morieri ML, Avogaro A, Fadini GP. The toll of lockdown against COVID-19 on diabetes outpatient care: Analysis from an outbreak area in northeast italy. Diabetes Care. American Diabetes Association Inc.; 2021. p. e18-21.

23. Izzo L, Santonastaso A, Cotticelli G, Federico A, Pacifico S, Castaldo L, et al. An Italian survey on dietary habits and changes during the COVID-19 lockdown. Nutrients. MDPI AG; 2021;13.

24. Celorio-Sardà R, Comas-Basté O, Latorre-Moratalla ML, ZerónRugerio MF, Urpi-Sarda M, Illán-Villanueva M, et al. Effect of COVID-19 Lockdown on Dietary Habits and Lifestyle of Food Science Students and Professionals from Spain. Nutrients. Nutrients; 2021;13.

25. Sidor A, Rzymski P. Dietary choices and habits during COVID19 lockdown: Experience from Poland. Nutrients. MDPI AG; 2020;12.

26. Tanji Y, Sawada S, Watanabe T, Mita T, Kobayashi Y, Murakami $\mathrm{T}$, et al. Impact of COVID-19 pandemic on glycemic control among outpatients with type 2 diabetes in Japan: A hospital-based 
survey from a country without lockdown. Diabetes Res Clin Pract. Diabetes Res Clin Pract; 2021;108840.

27. Rastogi A, Hiteshi P, Bhansali A. Improved glycemic control amongst people with long-standing diabetes during COVID-19 lockdown: a prospective, observational, nested cohort study. Int J Diabetes Dev Ctries. Springer; 2020;40:476-81.

28. Holmes EA, O'Connor RC, Perry VH, Tracey I, Wessely S, Arseneault L, et al. Multidisciplinary research priorities for the COVID-19 pandemic: a call for action for mental health science. The Lancet Psychiatry. Elsevier Ltd; 2020. p. 547-60.

29. Kornilaki EN. The psychological effect of COVID-19 quarantine on Greek young adults: Risk factors and the protective role of daily routine and altruism. Int J Psychol. Int J Psychol; 2021;

30. Chua BS, Siau CS, Fitriana M, Low W-Y, Abdul Wahab Khan RK Bin. Psychological Distress, Relationship Quality, and
Well-Being Among Malaysian Couples During the COVID-19 Pandemic. Asia-Pacific J public Heal. Asia Pac J Public Health; 2021;10105395211014322.

31. Pengo MF, Albini F, Guglielmi G, Mollica C, Soranna D, Zambra $\mathrm{G}$, et al. Home blood pressure during COVID-19-related lockdown in patients with hypertension. Eur J Prev Cardiol. 2021;

32. Fosco MJ, Silva P, Taborda GA, Ahumada L. Asociación entre aislamiento social preventivo obligatorio por COVID-19 e Hipertensión Arterial Grave. Medica. 2020;80:1-5.

Publisher's note Springer Nature remains neutral with regard to jurisdictional claims in published maps and institutional affiliations.

\section{Authors and Affiliations}

\section{Alev Selek ${ }^{1}\left[\right.$ Emre Gezer ${ }^{1} \cdot$ Eda Altun $^{2} \cdot$ Mehmet Sözen $^{1}$ [D $\cdot$ Ömercan Topaloğlu ${ }^{3} \cdot$ Damla Köksalan $^{1}$. Halil Demirkan $^{4} \cdot$ Dilek Karakaya $^{5} \cdot$ Berrin Cetinarslan $^{1} \cdot$ Zeynep Cantürk $^{1} \cdot$ Dilek Taymez $^{6}$}

\author{
Alev Selek \\ alevselek@gmail.com \\ Emre Gezer \\ gezeremre@gmail.com \\ Eda Altun \\ dredaaltun@hotmail.com \\ Ömercan Topaloğlu \\ drhomercan@hotmail.com \\ Damla Köksalan \\ drdamlakoksalan@gmail.com \\ Halil Demirkan \\ drhdk63@gmail.com \\ Dilek Karakaya \\ dilek.arpaci@medikalpark.com.tr \\ Berrin Cetinarslan \\ barslan@kocaeli.edu.tr \\ Zeynep Cantürk \\ zeynepcanturk@hotmal.com
}

Dilek Taymez

guvendilek@yahoo.com

1 Department of Endocrinology and Metabolism, Kocaeli University School of Medicine, Umuttepe, Kocaeli, Turkey

2 Department of Nephrology, Gölcük State Hospital, Kocaeli, Turkey

3 Department of Endocrinology and Metabolism, Derince Training and Research Hospital, Kocaeli, Turkey

4 Department of Endocrinology and Metabolism, Kocaeli SEKA State Hospital, Kocaeli, Turkey

5 Department of Endocrinology and Metabolism, Medical Park Hospital, Gebze, Kocaeli, Turkey

6 Department of Nephrology, Kocaeli State Hospital, Kocaeli, Turkey 